\title{
PREPARATION AND PROPERTIES OF CHITOSAN HYBRID FILMS FROM MICROWAVE IRRADIATED SOLUTIONS
}

\author{
S. FUENTES ${ }^{\prime}, J$. RETUERT ${ }^{2}$ AND G. GONZÁLEZ*3 \\ ${ }^{I}$ Department of Physics, Faculty of Sciences, Universidad Católica del Norte, Angamos 0610, Antofagasta, Chile. \\ ${ }^{2}$ Department of Material Sciences, Faculty of Mathematical and Physical Sciences, \\ Universidad de Chile; Tupper 2069, Santiago, Chile. \\ ${ }^{3}$ Department of Chemistry, Faculty of Sciences, Universidad de Chile, P.O. Box 653, Santiago, Chile. \\ (Received: 27 December 2007 - Accepted: 24 March 2008)
}

\begin{abstract}
The effect of microwave irradiation (MW, $1.55 \mathrm{~kW}$ at $2450 \mathrm{MHz}$ on the dissolution chitosan(CHI) and on the properties of the solutions was studied and the results compared with those obtained by using mechanical stirring. MW effects were tested by analysing the viscosity of the solutions as well as the properties of the films of the nanocomposite the chitosan with poly(3-(aminopropyl)triethoxysilane)(pAPS) they produce. Microwave irradiation induces rapid dissolution, leading to solutions with relatively low viscosity, which asymptotically increases with further irradiation. Dissolution-precipitation-redissolution experiments with samples prepared using both methods indicate that MW effects are more related with the aggregation state of the macromolecule in the solution than with the chitosan degradation. CHI/pAPS hybrid materials appear to be different when the solutions of chitosan used as precursor are obtained by MW irradiation. Thus, MW appears to affect the chitosan self-association so changing the equilibria between the micro domains involved in the macromolecular complex formation.
\end{abstract}

Keywords. Chitosan, Hybrid Films, Microwave.

BRIEFS. The dissolution procedure affects the degree of self-association of chitosan, which altering the packaging of the shackles of macromolecule in their supramolecular complexes influences on the properties of materials.

\section{INTRODUCTION}

Chitosan (CHI) is obtained by partial chitin N-deacetylation, poly-[(1-4)2-deoxy-2-acetamido-D-glucose], an abundant polysaccharide contained in crustacean exoskeletons (Schema 1). CHI is a copolymer composed mainly of $\beta$-(1-4)-2-amino-2-deoxy-D-glucopyranose units and, partially, of $\beta$-(1-4)-2amino-2-deoxy-2-acetamido-D-glucopyranose units ${ }^{1-2}$. This polysaccharide is structurally similar to cellulose, but the presence of the $-\mathrm{NH}_{2}$ amino group on the carbon- 2 of the chitosan unit drastically changes its properties by enhancing its solubility and reactivity. CHI-based products have numerous applications covering various fields like medicine, biotechnology, wastewater treatment, and artificial membranes, including artificial skin, among other uses ${ }^{3-4}$. The possibility of hydrogen bonding between CHI-amino groups and donor sites on inorganic networks, producing good compatibility of both phases, is particularly interesting.

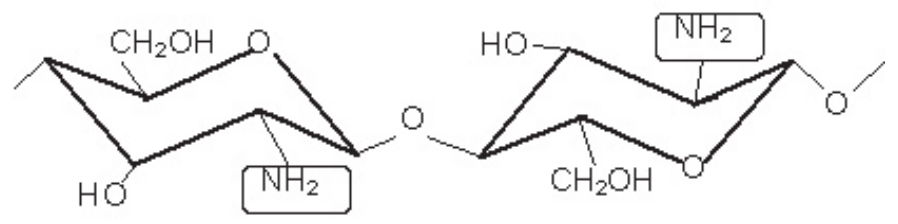

Scheme 1. Chemical structure of chitosan.

In nature, biological organisms often produce organic-inorganic hybrid materials like bone, teeth or shells which, compared to synthetic materials, have high-level mechanical properties; from this perspective, chitosan organicinorganic composites are particularly interesting ${ }^{5-8}$. The ability of chitosan to interact with inorganic molecules may be appreciated, for instance, in the formation of hybrid films of a Chilean chitosan (CHI-C) with poly(aminopro pylsiloxanes) (pAPS), pure or doped with lithium perchlorate, as reported by Fuentes et al. ${ }^{9-11}$. Experiments result in a well characterized CHI-C/pAPS hybrid materials which, because of the molecular compatibility between domains of the components, origine hybrid films with interesting thermal and optical properties. However, properties of the films depend on the aggregation state of the macromolecules in the solution which, in turn, is determined, not only by the particular chitosan with characteristic average molecular weight and acetylation degree, but also by the solvent and the method used for preparing the solutions. In general, any methodological or mechanistic change in the properties of the solutions of chitosan should be useful for regulating the properties of film they form, thus permitting to optimize the products for predetermined purposes. Microwave irradiation could be one of these methods.

In recent years microwave chemistry has received much attention ${ }^{12-15}$. It has been demonstrated that when chemical reactions are performed under microwave irradiation both, reaction rates and selectivity, may be significantly improved respect to those prepared under conventional conditions.

It is widely recognized that specific non-thermal effects of microwaves on organic substances are mostly related to molecular stirring produced by the disturbance of the molecule dipoles present in associated chemical systems, e.g. in those stabilized by hydrogen bonds ${ }^{16-19}$. MW radiation is therefore expected to be easily absorbed by solutions of chitosan that it is known to be strongly associated in solution. Indeed it has been reported that the solutions of chitosan always contain supramolecular aggregates which have between 2 and 8 macromolecule chains ${ }^{20}$. Since the degree of association determines the chain stiffness and, consequently, the viscosity and other properties of the $\mathrm{CHI}$ solutions, strong non-thermal effects of the microwave irradiation on these solutions are expected ${ }^{21}$.

In this paper is described how microwave radiation, during the dissolution of chitosan or in the treatments of its solutions, cause changes in the degree of self-association of this macromolecule, a method that could be useful in the desing and preparation of films of hybrid CHI-pAPS nanocomposites, thus providing an approach which not previously applied to the formation of these products.

\section{EXPERIMENTAL}

Materials. Chitosan, purchased from Aldrich (CHI-A), was washed with acetone and methanol and dried to constant weight. Its average molecular weight $\left(\mathrm{Mv}=3.5^{*} 10^{5}\right)$ was determined by light scattering as previously described $\mathrm{X}^{22}$. CHI-A acetylation degree (A.D) was estimated in $17 \%$.

Chitosan solutions: $1 \mathrm{~g}$ CHI-A was dissolved in 100-ml 5\% formic acid solutions by stirring about $24 \mathrm{~h}$ at room temperature. Resulting solutions were successively filtered through coarse- and fine-fritted glass and then microfiltered in a Millipore system using, consecutively, membranes with porosities of 3.0, 0.45 and $0.2-\mu \mathrm{m}$ repectively.

A solution containing about $0.7-\mathrm{g} / \mathrm{ml}$ aminopropylsiloxan (pAPS) partially polymerized was obtained by hydrolyzing 3-aminopropyltrietho xysilane (APS), Aldrich, in 0.5 -M formic acid at $45^{\circ} \mathrm{C}$ for three days. The solvent was removed by evaporation until the solution stopped flowing. After size exclusion chromatography analysis (Bruker LC 21B with a Shodex $\mathrm{OH}$ pack 803-column), obtained aminopropylsiloxane oligomers (pAPS) have an 
average molecular weight of about 800 .

MW-assisted CHI dissolutions. Small portions of CHI-A $(0.05 \mathrm{~g})$ were added to $5-\mathrm{ml} 5 \%$ formic acid solution and then put into a domestic microwave oven (SHARP R-450A, $1.55 \mathrm{~kW}$ at 2450-MHz frequency). A sequence of 5-s MW pulses was applied to the CHI suspension until complete dissolution $(660$ s). In order to avoid excess heating, temperature of the sample was controlled $\left(<40{ }^{\circ} \mathrm{C}\right)$. Thereafter, the solutions were filtered as above.

Film preparation. Two series of films, using both $\mathrm{CHI}$ dissolved by mechanical stirring (MS) and microwave irradiation (MW) respectively, were prepared. In both cases, CHI-A and pAPS solutions were mixed in proper amounts to get $\mathrm{CHI} / \mathrm{pAPS}$ 1:1 molar ratios and then stirred at room temperature for $24 \mathrm{~h}$. Films were then prepared by solution casting on a polypropylene film. The solvent was evaporated at room temperature. Varying the solution concentration, 50 to $100 \mu \mathrm{m}$-thick films were prepared.

Analytical methods. Viscosity changes during the reaction were monitored by falling-ball viscosimetry. The average viscosimetric molecular weight of chitosan was estimated from its intrinsic viscosity determined in the solvent $0.1 \mathrm{M} \mathrm{CH}_{3} \mathrm{COOH} / 0.2 \mathrm{M} \mathrm{NaCl}$ using the Mark-Houwink parameters $a=0.96$ and $K_{n}=1.424$ at $25^{\circ} \mathrm{C}$.

Characterization. Films were characterized by simultaneous thermal analysis, DTA/TGA (STA 409 NETZSCH), FT-IR spectra (Perkin Elmer 2000) and X-ray diffraction (Siemens D-500). Morphological characterization was done by Scanning Electron Microscopy (Philips EM 300).

\section{RESULTS AND DISCUSSION}

\section{Chitosan Dissolution and re-precipitation.}

In Table 1 are compared the viscosity of CHI-A solutions obtained by the two methods, MW-irradiation and mechanical stirring (MS). In the same table are also indicated the respective viscosities of the solutions after a reprecipitation process. As observed in entry 1 , the viscosity of the solution of chitosan dissolved using MW is significantly lower than that using MS, 171 and $341 \mathrm{cp}$ respectively.

The experiments described in the entries 2 and 3 of Table 1 were performed in order to elucidate if the MW assisted process is affecting only inter-chain associations or if it is producing chain degradation. In these experiments samples of solutions obtained by both methods are precipitated by rapid addition of alkali and then re-dissolved by MS. If the dissolution assisted by MW-irradiation produces a degradation of the sugar chains in a greater extent than that performed by mechanical stirring, the viscosities of both solutions after a precipitation-thermal re-dissolution cycle have to much different. A significantly lower viscosity for the chitosane originally dissolved under MWirradiation would be indeed expected. However, as may be seen in Table 1, the viscosity values in both cases are practically the same. Moreover, when these solutions are further irradiated both show a similar behavior. Thus, from the point of view of the viscosity of re-dissolved chitosane, the precipitation method appears to be relatively more important than the procedure used for obtaining pristine solutions. That is corroborated by the study described in Figure 1, which shows the effect of time of irradiation on the viscosity after the first dissolution. The samples of both, MW-assisted and MS obtained solutions, were stepwise irradiated with $660 \mathrm{~s}$ doses, until a total irradiation of $9900 \mathrm{~s}$. As observed, changes appear to follow approximately the same law in both cases. Differences are indeed determined only by the magnitude of the effect caused by the dissolution process then it can be conclude that the dissolution process essentially consists in producing soluble supramolecular aggregates by the partial rupture of intermolecular hydrogen bonds. The magnitude of this process and thus the average size of the aggregates in the solutions is however strongly influenced by the method employed for dissolving, being in our case much more effective the action of the microwave radiation than that of the mechanical stirring.
Table 1. Falling-ball viscosity at $25{ }^{\circ} \mathrm{C}$ of chitosan solutions prepared under different experimental conditions.

\begin{tabular}{clcc}
\hline Entry & Sample Treatment & $\begin{array}{c}\text { Sample } \\
\text { dissolved by } \\
\text { MW }\end{array}$ & $\begin{array}{c}\text { Sample } \\
\text { dissolved by } \\
\text { MS }\end{array}$ \\
\hline 1 & $\begin{array}{l}\text { Initial Viscosity } \\
\text { Viscosity of the samples } \\
\text { after precipitating them and } \\
\text { re-dissolved by mechanical } \\
\text { stirring }\end{array}$ & $78[\mathrm{cp}]$ & $341[\mathrm{cp}]$ \\
& $\begin{array}{l}\text { Viscosity of the samples } \\
\text { after radiating them with } \\
\text { microwave }(660 \mathrm{~s}) .\end{array}$ & $58[\mathrm{cp}]$ \\
\hline
\end{tabular}

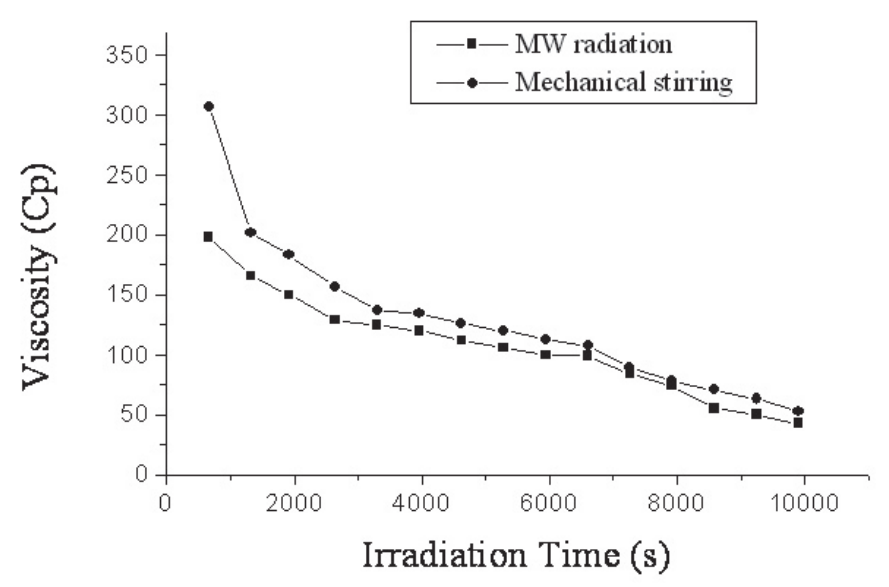

Figure 1. Effect of the MW irradiation dose on the viscosity of chitosan solutions obtained by $\left(-\bullet_{-}\right)$mechanical stirring and $\left(--_{-}\right)$MW irradiation. Samples were consecutively radiated from $660 \mathrm{~s}$ to $9900 \mathrm{~s}$.

The different extend of the molecular interactions in the chitosane a solution, which as discussed above depends on dissolution procedure, MWirradiation or mechanical stirring, may be also detected in the properties of the films prepared from both type of solutions. As observed in Figure 2, the thermal stability of these two solids is clearly different. Films prepared using solutions obtained by MW-irradiation result to be somewhat more stable. The temperatures of decomposition, though within the range characteristic for the decomposition of chitosan, are different, similar to $300{ }^{\circ} \mathrm{C}$ and 282 ${ }^{\circ} \mathrm{C}$ respectively. These results confirm the hypothesis above; i.e., MW energy produces solutions containing relatively smaller aggregates, what allows better intermolecular matching during the formation of the solid phase, thus increasing the thermal stability of the film. 


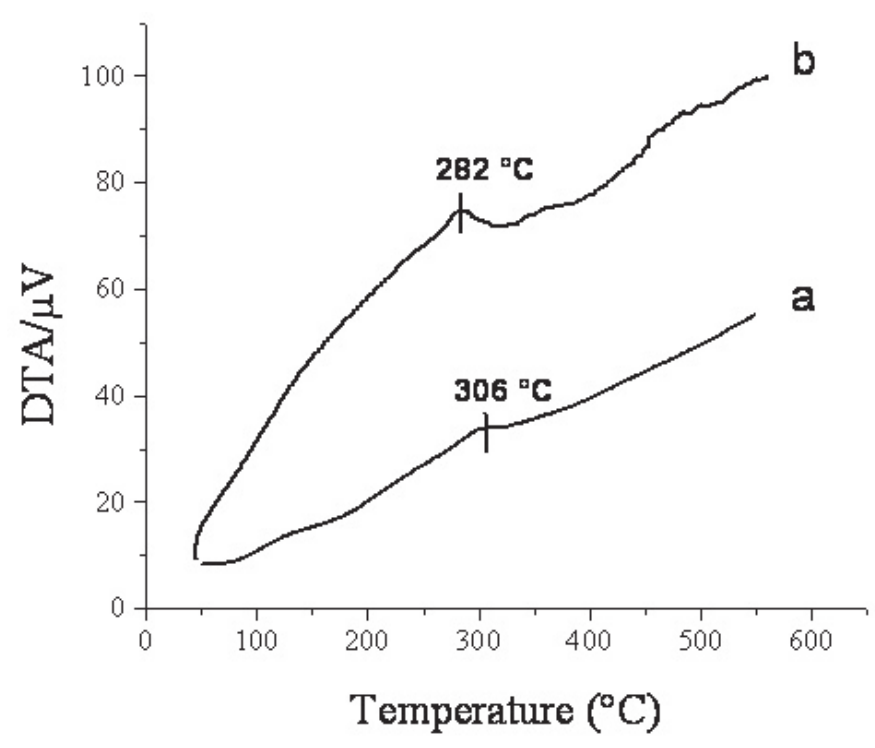

Figure 2. Differential Thermal Analysis of chitosan films obtained from solutions generated by (a) microwave and (b) mechanical stirring dissolutionassisted processes.

\section{Chitosan- p(aminopropyl Siloxane) nanocomposites.}

In the chitosan there are hydroxyl- and amino-groups which allow it to interact with other species forming supramolecular entities stabilized by hydrogen bonding and/or Lewis acid-base interactions which often lead to interphases in a nanometer scale. The formation of these nanocomposites often improves the properties of the pristine polysaccharide. An example of such nanocomposites is the macromolecular complex formed by the Chilean chitosane (CHI-C) with poly(aminopropylsiloxane) (pAPS) previously informed by us $^{9-11}$, which with a stoichiometry CHI-C/pAPS 0.6:1 origins stable, transparent films. CHI-A also forms a macromolecular complex but with a stoichiometry ratio $\mathrm{CHI}-\mathrm{A} / \mathrm{pAPS} 1: 1$; i.e., a higher amount of chitosan is needed to obtain the molecularly compatible microdomains responsible for the transparency of the material. This difference may be rationalized considering the somewhat lower deacetilation degree of CHI-A which favors chitosan self-association. That not withstanding, we have found that the properties of $\mathrm{CHI}-\mathrm{A} / \mathrm{pAPS}$ nanocomposites may be altered by the MW irradiation of the precursor solutions.

In Figure 3 are compared the thermal stabilities of the CHI-A/pAPS complexes with that of the pure chitosan. The decomposition temperature of the polymer complex prepared using the polysaccharide dissolved by mechanical stirring is $38^{\circ} \mathrm{C}$ higher than that of the pristine polymer, while this difference reaches only $20^{\circ} \mathrm{C}$ in the case of the polysaccharide previously treated with MW radiation. These characteristics can be explained by the tendency of irradiated solutions to promote free association in the formation of the films mentioned above, which, in this case, leads to an improvement in the chi-chi interchain interactions at the expense of chi-p APS association.

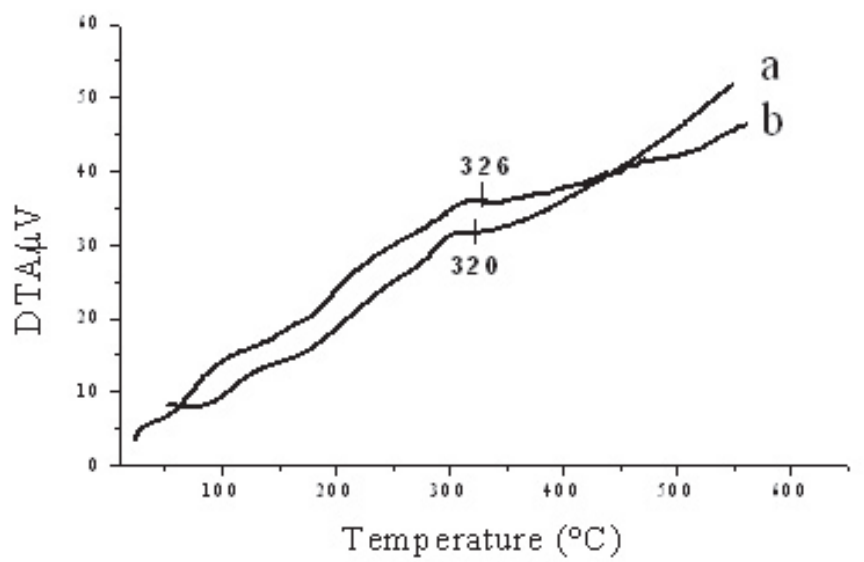

Figure 3. Influence of the dissolution method on pure CHI-A DTA and its APS complex: (a) CHI-A/pAPS 1:1 (MW), and (b) CHI-A/pAPS 1:1 (MS).

The effect of the MW is also apparent in the microstructure of the products. As can be seen in Figure 4a, the surface is no more regularly globular and the formation of cracks is observed, contrasting with the surface of films obtained starting from CHI dissolved by mechanical stirring (Figure 4b). That is again a manifestation of the enhanced tendency to auto-association induced by the MW irradiation of the precursor solution, which prevents the formation of strong interphases with the pAPS.
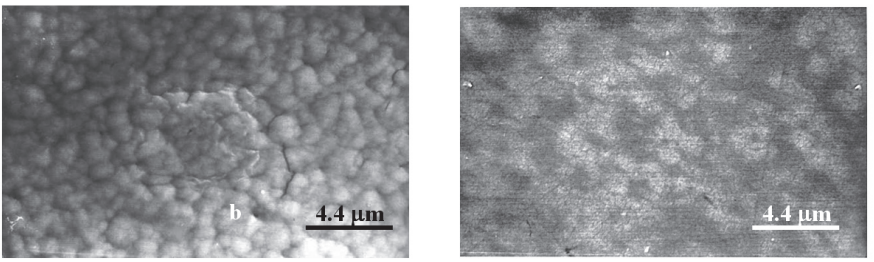

Figure 4. SEM micrographs of 1:1 CHI-A/pAPS films prepared with $\mathrm{CHI}$ dissolved by: (a) Mechanical stirring (MS), and (b) Microwave irradiation.

\section{CONCLUSIONS}

Results discussed above show that the aggregation state of chitosan in its solutions, strongly influenced by dissolution method, is an important variable for determining the properties of chitosan-based materials. Microwave irradiation results in an interesting method for changing chitosan macromolecule selfassociation degree. Films and composite materials obtained from irradiated solutions show thermal and morphological behaviors different from those using solutions obtained by conventional methods. In general, MW-irradiated solutions produce solid phases in which the formation of polysaccharide network by hydrogen bonding appears to be favored. Thus the use of different MW irradiation doses could be useful in order to regulate, to some extent, the properties of the products.

Finally, results show that by properly combining variables, such as microwave irradiation treatment of solutions and/or the use additives, it is possible to obtain tailor-made chitosan-based products useful for specific applications.

\section{ACKNOWLEDGMENT}

Research partially funded by FONDECYT 1050344. 


\section{REFERENCES}

1. Chandy, T., Sharma, C. P. Biomater. Artif. Cells Artif. Organs. 18, 1 (1990).

2. Robert, G. A. Chitin Chemistry; Macmillan Press: 1992, London.

3. N. Boucard, C. Viton, D. Agay, E. Mari, T. Roger, Y. Chancerelle and A. Domard. Biomaterials. 28, 3478 (2007).

4. Majeti N. V. Ravi Kumar. React. and Funct. Polym. 46, 27 (2000).

5. Y. Martínez, J. Retuert, M. Yazdani-Pedram and H. Cölfen. Polym. 45, 325 (2004).

6. H. Jiankang, L. Dichen, L. Yaxiong, Y. Bo, L. Bingheng and L. Qin. Polym. 48, 4578 (2007).

7. S. M. Lai, A. Yang, W. Chen, et al. Polym. Plast. Technol. Eng. 45 (9), 997 (2006).

8. F. Mammeri, E. Le Bourhis, L. Rozes, et al. J. Mater. Chem. 15 (35), 3787 (2005).

9. S. Fuentes, P. J. Retuert, A. Ubilla, J. Fernández and G. González. Biomacromolecules. 1, 239 (2000).

10. S. Fuentes, P. J. Retuert and G. González. Electrochem. Acta. 48, 2015 (2003).
11. S. Fuentes, P. J. Retuert, G. González, E.Ruiz-Hitzky. International J. Polymeric Mater. 35, 61 (1997).

12. R. Hoogenboom, U. Schubert. Macromol. Rapid Commun. 28, 368 (2007).

13. F. Wiesbrock, R. Hoogenboom, U. Schubert. Macromol. Rapid Commun. 25, 1739 (2004)

14. D. Dallinger, C. Kappe Chemical Reviews. 107, 2563 (2007).

15. R. Hoogenboom, M. Leenen, F. Wiesbrock, et al. Macromol. Rapid Commun. 26, 1773 (2005).

16. A de la Hoz, A. Az-Ortiz, A. Moreno, Chem. Soc. Rev. 34, 164 (2005).

17. C. O. Kappe, Angew. Chem. Int. Ed. 43, 6250 (2004).

18. P. Lidstrom, J. Tierney, B. Wathey, J. Westman, Tetrahedron. 57, 9225 (2001).

19. D. Bogdal, P. Penczek, J. Pielichowski, A. Prociak, Adv. Polym. Sci. 163, 193 (2003).

20. Salmon, S.; Hudson, S. Rev. Macromol. Chem. Phys., C37, 199 (1997).

21. M. Tsaih, R. Chen. Int. J. Biol. Macromol. 20, 233 (1997).

22. M. Rinaudo, P. Le Dung, M. Millas. Int. J. Biol. Macromol. 15, 281 (1993). 